\title{
Estimação da área foliar por método não destrutivo, utilizando medidas lineares das folhas de espécies de Passiflora ${ }^{1}$
}

\author{
Marcos Antonio Dell'Orto Morgado 2 , Claudio Horst Bruckner ${ }^{3}$, Luciana Domiciano Silva Rosado ${ }^{4}$ \\ Welberth Assunção ${ }^{5}$, Carlos Eduardo Magalhães dos Santos ${ }^{3}$
}

\begin{abstract}
RESUMO
Objetivou-se, com este trabalho, desenvolver uma equação confiável, que possibilite calcular a área foliar de forma rápida e não destrutiva, utilizando medidas lineares da folha, para as diferentes espécies de Passiflora ( $P$. alata, $P$. coccinea, . gibertii, . ligularis, . misera, $P$. mucronata, $P$. nitida, . setacea). Foram coletadas trezentas folhas, de diferentes tamanhos, de cada espécie. Realizaram-se análises de regressão da área foliar versus comprimento da nervura principal, maior largura da folha e o produto destas. A área foliar foi medida com um dispositivo de medição automática e as medidas lineares foram determinadas utilizando-se régua. O modelo linear escolhido, para todas as espécies, foi o que utiliza o produto das variáveis independentes, por apresentar maior coeficiente de determinação $\left(\mathrm{R}^{2}\right)$ e maior significância do coeficiente de regressão. Os modelos apresentaram coeficiente de determinação ajustado superior a 0,927 e índice de desempenho ótimo, segundo a classificação de Camargo e Sentelhas. No processo de validação do modelo, mostrou-se que a correlação da área foliar medida com a área foliar observada foi muito alta. Os resultados obtidos neste estudo demonstram que a área foliar das espécies de Passifloras podem ser preditas, usandose as determinações do comprimento e da largura das folhas.
\end{abstract}

Palavras-chave: maracujá, medidas lineares, Passifloracea, validação de modelos.

\section{ABSTRACT}

\section{Estimation of leaf area by non-destructive method using linear measurements of leaves of Passiflora species}

The objective of the present study was to develop a reliable equation which enables a fast and non-destructive calculation of leaf area using linear leaf measures from different Passiflora species ( $P$. alata, $P$. coccinea, $P$. gibertii, $P$. ligularis, $P$. misera, $P$. mucronata, $P$. nitida, . setacea). Three hundred leaves with different sizes were collected from each species. It was performed the analysis of regression of leaf area versus lenght of the main vein, greatest leaf width and their product. Leaf area was measured with a device for automatic measurement and linear measures were determined using a ruler. The linear model chosen for all species, was the one which uses independent variables product because it presents the greatest coefficient of determination $\left(\mathrm{R}^{2}\right)$ and significative coefficient of regression. The models showed adjusted coefficient of determination higher than 0.927 and optimal performance index, according to the classification of Camargo and Sentelhas. In the process of model validation, it was shown that the correlation between measured leaf area and observed leaf area was very high. The results of this study demostrate that leaf area of Passiflora species can be predicted, using the measurements of the length and width of leaves.

Key words: passion fruit, linear measures, Passifloracea, model validation.

\footnotetext{
Recebido para publicação em 19/12/2012 e aprovado em 27/06/2013.

${ }^{1}$ Extraído da tese de doutorado do primeiro autor.

${ }^{2}$ Engenheiro-Agrônomo, Doutor. Instituto Federal do Espírito Santo, Campus Itapina, Rodovia BR 529, Km 70, Zona Rural, 29709-910, Colatina, Espírito Santo, Brasil. agrodellorto@yahoo.com.br

${ }^{3}$ Engenheiro-Agrônomo, Doutor. Departamento de Fitotecnia, Universidade Federal de Viçosa, Campus Viçosa, Avenida Peter Henry Rolfs, s/n, 36570-000, Viçosa, Minas Gerais, Brasil. bruckner@ufv.br; carlos.magalhaes@ufv.br (autor para correspondência).

${ }^{4}$ Engenheira-Agrônoma, Doutora. Departamento de Fitotecnia, Universidade Federal de Viçosa, Campus Viçosa, Avenida Peter Henry Rolfs, s/n, 36570-000, Viçosa, Minas Gerais, Brasil. luciana.rosado@ufv.br

${ }^{5}$ Engenheiro-Agrônomo. Departamento de Fitotecnia, Universidade Federal de Viçosa, Campus Viçosa, Avenida Peter Henry Rolfs, s/n, 36570-000, Viçosa, Minas Gerais, Brasil. welberth_assuncao@ hotmail.com
} 


\section{INTRODUÇÃO}

As espécies de maracujá (Passiflora spp.), pertencente à família Passifloraceae, ocorrem praticamente em todo o território brasileiro; entretanto, poucas delas são caracterizadas. A escassez de informações denota a necessidade de estudos que vão desde o simples levantamento do número de espécies que ocorrem em uma determinada localização, até a obtenção de informações sobre seu sistema reprodutivo, morfologia e análise genômica.

Estudos com espécies silvestres constituem importante alternativa para usos de novos genótipos no melhoramento das espécies cultivadas comercialmente (Ataíde et al., 2012), com incorporação de características de interesse.

A análise quantitativa do crescimento é o primeiro passo na avaliação da produção vegetal e requer informações que podem ser obtidas sem a necessidade de equipamentos sofisticados. O monitoramento da área foliar é uma ferramenta importante para estudar as características fisiológicas relacionadas com o crescimento das plantas, as relações fotossintéticas e os processos de transpiração, bem como, são um índice útil na avaliação de danos causados por doenças e pragas foliares (Monteiro et al., 2005; Favarin et al., 2002).

Na cultura do maracujazeiro, a determinação da área foliar proporciona fundamentação aos estudos fisiológicos, que envolvam o crescimento da planta, a interceptação de luz, a eficiência fotossintética, a evapotranspiração e a resposta a fertilizantes e à irrigação (Blanco \& Follegatti, 2005). A folha é o principal órgão no processo transpiratório, responsável pelas trocas gasosas entre a planta e o ambiente. Por esta razão, compreender o crescimento e o desenvolvimento do maracujazeiro possibilita a implantação de estratégias de manejo para o alto rendimento. A obtenção de medidas precisas da área foliar é essencial para o entendimento da interação entre o crescimento da cultura e seu ambiente (Jesus et al., 2001).

Vários métodos são usados para estimar a área foliar (Lu et al., 2004), sendo que o mais comum é estimar a área foliar por equações matemáticas, envolvendo medidas lineares como comprimento e largura da folha e comprimento do pecíolo, ou alguma combinação dessas variáveis, o que geralmente têm apresentado alta precisão, como com pepino (Blanco \& Folegatti, 2005) e meloeiro (Lopes et al., 2007).

Esses métodos usualmente são rápidos e não destrutivos, têm apresentado boa precisão e alta acurácia (Campostrini \& Yamanishi, 2001; Bianco et al., 2003), além de permitirem a realização de medidas repetidas ao longo do período de desenvolvimento da planta, e reduzirem, assim, a variabilidade associada com procedimentos de amostragens destrutivas (Nesmith, 1992), como apresentado para as culturas da alface (Guo \& Sun, 2001), tomate (Astegiano et al., 2001) e pepino (Blanco \& Folegatti, 2005; Cho et al., 2007).

A determinação de uma metodologia de medição de área foliar de baixo custo, eficiente, rápida e não destrutiva, é imprescindível para a ampliação das pesquisas com as espécies de Passiflora. Deste modo, objetivou-se produzir uma equação confiável que estime a área foliar de plantas de maracujá, de forma não destrutiva, utilizando medidas lineares das folhas como variáveis independentes, para as diferentes espécies de Passiflora.

\section{MATERIAL E MÉTODOS}

Foi conduzido um experimento com maracujazeiro, no campo experimental pertencente à Universidade Federal de Viçosa (UFV), Viçosa, MG [ $20^{\circ} 45^{\prime} \mathrm{S}, 42^{\circ} 52^{\prime} \mathrm{O}$, altitude 648 m, clima do tipo Cwa - clima mesotérmico (Köppen), precipitação média anual de 1.200 mm e temperatura média anual de $\left.19^{\circ} \mathrm{C}\right]$.

As mudas de oito espécies do gênero Passiflora ( $P$. alata, $P$. coccinea, $P$. gibertii, $P$. ligularis, $P$. misera, $P$. mucronata, $P$. nitida, $P$. setacea) foram transplantadas em março de 2009, utilizando-se o espaçamento de 4,0 m entre plantas e 3,5 m entre linhas, realizando-se os tratos culturais preconizados para a cultura do maracujazeiro azedo. Transcorridos quinze meses após o transplantio, foram selecionadas aleatoriamente 300 folhas por espécie, padronizando-se as amostras por formato e tamanho. Essas folhas foram cuidadosamente destacadas e transportadas em caixas térmicas ao laboratório, onde se mensurou a área foliar com medidor de área foliar de bancada. As folhas selecionadas não apresentavam qualquer dano ou deformação causada por doença, insetos ou outros fatores externos.

A morfologia foliar é variável entre as espécies, podendo ser desde oblongo-ovaladas a trilobadas. Sendo assim, em espécies com folhas inteiras (não lobadas) foram mensurados o comprimento da nervura principal (c) e a maior largura da folha (1). Em espécies que possuem mais de um lóbulo por folha, foram mensurados o comprimento da nervura principal (C) e a maior largura entre a extremidade distal dos lóbulos mais externos (L), conforme ilustrado na Figura 1. Estas medições foram feitas utilizando-se régua com precisão de $0,1 \mathrm{~cm}$.

Para cada espécie, foi modelada a relação entre a área foliar de cada folha com as dimensões lineares de comprimento (c ou C ) e largura (1 ou L) das folhas ou o produto entre estas variáveis, em decorrência da morfologia, por meio dos modelos: $y=\beta_{0}+\beta_{1}(\mathrm{c}$ ou $\mathrm{C}) ; \mathrm{y}=\beta_{0}+\beta_{1}(\mathrm{lou} \mathrm{L}) ; \mathrm{y}$ $=\beta_{0}+\beta_{1}(\mathrm{cl}$ ou $\mathrm{CL})$ e $\mathrm{y}=\beta_{0}+\beta_{1}(\mathrm{c}$ ou $\mathrm{C})+\beta_{2}(1$ ou $\mathrm{L})$, em que y representa a variável dependente observada; $\beta_{0}, \beta_{1}$ e $\beta_{2}$, 
os parâmetros a serem estimados; c ou C representa a variável independente comprimento; e 1 ou L representa a variável independente largura. Das 300 folhas mensuradas de cada espécie, 250 foram utilizadas para o desenvolvimento da equação de regressão. Para avaliar estatisticamente o desempenho dos modelos desenvolvidos, para estimar a área foliar das diferentes espécies, utilizaram-se 50 folhas com intuito de correlacionar os valores medidos com os estimados, tomando-se como base os indicadores estatísticos propostos por Camargo \& Sentelhas (1997), definidos da seguinte forma: precisão - coeficiente de correlação "r"; exatidão - índice de Willmott "d" e de confiança ou desempenho "c".

$\mathrm{O}$ coeficiente de correlação (r) permite quantificar o grau de associação entre as duas variáveis envolvidas na análise, sendo um indicativo de precisão do modelo.

O índice de concordância (d) fornece o grau de exatidão entre as variáveis envolvidas, uma vez que está relacionada com as diferenças entre os valores estimados e os valores observados, sendo seu campo de variação de 0 (nenhuma concordância) a 1 (concordância perfeita) (Willmott et al., 1985). O índice de concordância foi calculado pela expressão:

$\mathrm{d}=1-\left[\sum(\mathrm{Ae}-\mathrm{Ar})^{2} / \sum(|\mathrm{Ae}-\overline{\mathrm{Ar}}|+|\mathrm{Ar}-\overline{\mathrm{Ar}}|)^{2}\right]$

em que, Ae são os valores da área foliar estimada pela equação desenvolvida, Ar são os valores observados da área foliar, $\overline{\mathrm{Ar}}$ média dos valores da área real da folha.

Em seguida, obteve-se o índice de confiança ou desempenho "c", o qual indica o desempenho dos métodos (modelos), segundo Camargo \& Sentelhas (1997), sendo calculado pelo produto dos índices de precisão " $r$ " e de exatidão "d":

"c " $=\mathrm{r} * \mathrm{~d}$

O critério adotado para interpretação do desempenho dos modelos pelo índice "c" é apresentado na Tabela 1.

As análises de regressão foram realizadas com o programa SAS, (SAS Institute, 2004).

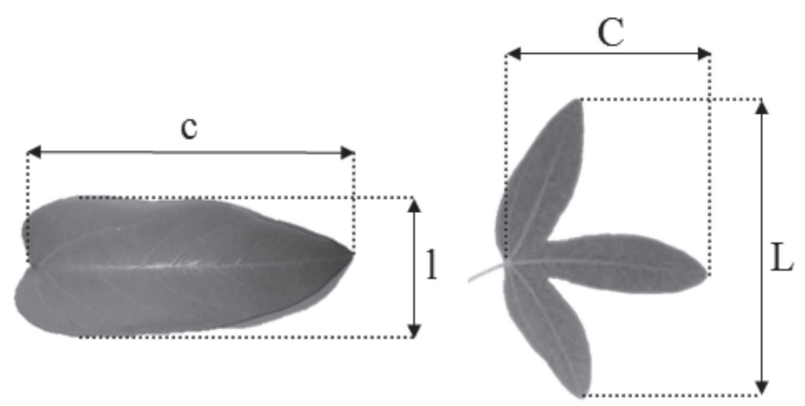

Figura 1. Partes medidas nas folhas de Passifloráceas para determinar o comprimento da nervura principal $(\mathrm{C}, \mathrm{c})$ e a maior largura da folha $(\mathrm{L}, 1)$.

\section{RESULTADOS E DISCUSSÃO}

A utilização das dimensões lineares comprimento e largura proporcionou uma estimativa satisfatória da área foliar, para as diferentes espécies de Passiflora. Na Tabela 2, estão apresentados os resultados da análise de regressão para a estimativa da área foliar. Estimaram-se quatro modelos para cada espécie estudada e, de maneira geral, os modelos linear e quadrático, que relacionam comprimento (C ou c), largura (L ou l) e o produto entre ambas com a área foliar determinada (Y), apresentaram elevados coeficientes de determinação $\left(R^{2} \geq 0,772\right)$.

Estimativas de coeficientes de determinação superiores a 0,92 foram encontrados por Cargnelutti Filho et al. (2012) e Toebe et al. (2010) para as culturas da mucunacinza e crambe, respectivamente, superiores a alguns encontrados neste trabalho para determinados modelos.

Ao se analisarem os coeficientes de determinação e a significância dos coeficientes de regressão $\left(\beta_{1}\right.$ ou $\left.\beta_{2}\right)$, verificou-se maior precisão do modelo que utilizou o produto entre as dimensões comprimento e largura na estimação da área foliar, optando-se por este modelo para a determinação da área foliar das espécies de Passiflora. Consequentemente, a adoção deste modelo possibilita a estimação da área foliar das espécies silvestres estudadas, com elevada precisão, necessitando-se apenas da mensuração do comprimento da nervura principal e da maior largura da folha ou da maior largura entre a extremidade distal dos lóbulos mais externos.

A área foliar é, comumente, amostrada em experimentos envolvendo fisiologia de fruteiras e produtividade de hortaliças, em que alguns fenômenos fisiológicos, como a fotossíntese, a respiração, o consumo de água e a transpiração têm merecido destaque, por estarem diretamente associados à área foliar (Centritto et al., 2000). Além das diferentes taxas de fotossíntese e transpiração, a área foliar também afeta a taxa de crescimento e a qualidade e produção de frutos do maracujazeiro.

A relação entre a área foliar e as dimensões foliares tem sido estabelecida para diversas espécies de plantas, como kiwi (Uzun \& Çelik,1999), morango (Demirsoy et al., 2005) e uva (Uzun \& Çelik, 1999), apresentando, esses

Tabela 1. Faixas de classificação do índice de confiança "c" com respectiva interpretação do desempenho

\begin{tabular}{ll}
\hline Valor de "c" & Interpretação \\
\hline$>0,85$ & Ótimo \\
0,76 a 0,85 & Muito bom \\
0,66 a 0,75 & Bom \\
0,61 a 0,65 & Mediano \\
0,51 a 0,60 & Sofrível \\
0,41 a 0,50 & Mal \\
$<0,40$ & Péssimo \\
\hline
\end{tabular}


autores, a existência de relação entre a área foliar com o produto entre o comprimento da nervura principal e largura da folha (com $\mathrm{R}^{2}$ variando de 0,95 a 0,98 ).

As cinquenta folhas de cada espécie, não utilizadas para gerar os modelos em estudo, foram utilizadas para realizar uma comparação entre a área foliar mensurada e a área foliar estimada pelo modelo escolhido neste trabalho. O objetivo desta comparação foi determinar o grau de acurácia do modelo (Figura 2). Verificou-se que o coeficiente de determinação $\left(R^{2}\right)$ entre a área foliar observada e a estimada foi superior a 0,929, para todas as espécies.

Utilizando-se o critério de interpretação do desempenho dos modelos pelos indicadores estatísticos, proposto por Camargo \& Sentelhas (1997) (Tabela 01), pode-se afirmar que os modelos propostos para estimar a área foliar por meio da medida de dimensões lineares da folha apre-

Tabela 2. Estimativas de coeficiente linear $\left(\beta_{0}\right)$, do angular $\left(\beta_{1}\right.$ e $\left.\beta_{2}\right)$ e respectivos erros padrão (entre parênteses), valor calculado do F e do coeficiente de determinação ajustado $\left(R^{2}\right)$, obtidos no ajuste do modelo de regressão entre a área foliar (variável dependente) e as dimensões lineares de comprimento (c ou C) e largura (l ou L) de folhas de oito espécies de maracujazeiro

\begin{tabular}{|c|c|c|c|c|c|}
\hline P. alata & $\beta_{0}$ & $\beta_{1}$ & $\beta_{2}$ & $\mathbf{F}_{\text {calc }}$ & $\mathbf{R}_{\text {ajust. }}^{2}$ \\
\hline Área $=\beta_{0}+\beta_{1}(\mathrm{c})$ & $-84,9\left(4,70^{a}\right)$ & $13,1(0,38)^{* *}$ & - & 1186,55 & 0,891 \\
\hline Área $=\beta_{0}+\beta_{1}(1)$ & $-57,0(2,40)$ & $16,73(0,30)^{* *}$ & - & 3057,61 & 0,955 \\
\hline Área $=\beta_{0}+\beta_{1}(\mathrm{cl})$ & $-3,7(0,80)$ & $0,78(<0,01)^{* *}$ & - & 10284,10 & 0,986 \\
\hline Área $=\beta_{0}+\beta_{1}(\mathrm{c})+\beta_{2}(1)$ & $-71,0(2,50)$ & $4,27(0,49)^{* *}$ & $11,91(0,61)^{* *}$ & 2349,14 & 0,970 \\
\hline P. coccinea & $\beta_{0}$ & $\beta_{1}$ & $\beta_{2}$ & $\mathbf{F}_{\text {calc }}$ & $\mathbf{R}_{\text {ajust. }}^{2}$ \\
\hline Área $=\beta_{0}+\beta_{1}(\mathrm{c})$ & $-62,9(4,63)$ & $9,97(0,39)^{* *}$ & - & 652,78 & 0,815 \\
\hline Área $=\beta_{0}+\beta_{1}(1)$ & $-35,2(2,16)$ & $16,28(0,38)^{* *}$ & - & 1785,82 & 0,923 \\
\hline Área $=\beta_{0}+\beta_{1}(\mathrm{cl})$ & $-0,7(0,50)$ & $0,82(<0,01)^{* *}$ & - & 13555,6 & 0,989 \\
\hline Área $=\beta_{0}+\beta_{1}(\mathrm{c})+\beta_{2}(1)$ & $-54,64(1,96)$ & $3,98(0,28)^{* *}$ & $11,33(0,429) * *$ & 2207,81 & 0,968 \\
\hline P. giberti & $\beta_{0}$ & $\beta_{1}$ & $\boldsymbol{\beta}_{2}$ & $\mathbf{F}_{\text {calc }}$ & $\mathbf{R}_{\text {ajust. }}^{2}$ \\
\hline Área $=\beta_{0}+\beta_{1}(\mathrm{C})$ & $-33,1(1,40)$ & $8,92(0,22)^{* *}$ & - & 1629,40 & 0,921 \\
\hline Área $=\beta_{0}+\beta_{1}(\mathrm{~L})$ & $-30,2(1,45)$ & $5,77(0,15)^{* *}$ & - & 1376,09 & 0,908 \\
\hline Área $=\beta_{0}+\beta_{1}(\mathrm{CL})$ & $-2,0(0,64)$ & $0,41(<0,01)^{* *}$ & - & 1773,97 & 0,927 \\
\hline Área $=\beta_{0}+\beta_{1}(\mathrm{C})+\beta_{2}(\mathrm{~L})$ & $-33,22(1,23)$ & $5,17(0,60)^{* *}$ & $2,58(0,39)^{* *}$ & 1081,23 & 0,940 \\
\hline P. ligulares & $\beta_{0}$ & $\beta_{1}$ & $\beta_{2}$ & $\mathbf{F}_{\text {calc }}$ & $\mathbf{R}_{\text {ajust. }}^{2}$ \\
\hline Área $=\beta_{0}+\beta_{1}(\mathrm{c})$ & $-134,69(9,37)$ & $21,03(0,71)^{* *}$ & - & 873,96 & 0,855 \\
\hline Área $=\beta_{0}+\beta_{1}(1)$ & $-107,39(5,54)$ & $20,35(0,43)^{* *}$ & - & 2211,62 & 0,937 \\
\hline Área $=\beta_{0}+\beta_{1}(\mathrm{cl})$ & $-4,98(2,79)$ & $0,88(0,02)^{* *}$ & - & 3022,40 & 0,953 \\
\hline Área $=\beta_{0}+\beta_{1}(\mathrm{c})+\beta_{2}(1)$ & $-122,44(5,76)$ & $5,45(1,07)^{* *}$ & $15,74(0,99)^{* *}$ & 1303,77 & 0,947 \\
\hline P. misera & $\beta_{0}$ & $\beta_{1}$ & $\boldsymbol{\beta}_{2}$ & $\mathbf{F}_{\text {calc }}$ & $\mathbf{R}_{\text {ajust. }}^{2}$ \\
\hline$\widehat{A r e a}=\beta_{0}+\beta_{1}(\mathrm{C})$ & $-19,42\left(2,30^{a}\right)$ & $11,18(0,61)^{* *}$ & - & 331,97 & 0,772 \\
\hline Área $=\beta_{0}+\beta_{1}(\mathrm{~L})$ & $-18,26(1,57)$ & $4,66(0,18)^{* *}$ & - & 676,23 & 0,873 \\
\hline Área $=\beta_{0}+\beta_{1}(\mathrm{CL})$ & $-1,94(0,64)$ & $0,72(0,02)^{* *}$ & - & 1576,16 & 0,941 \\
\hline Área $=\beta_{0}+\beta_{1}(\mathrm{C})+\beta_{2}(\mathrm{~L})$ & $-23,07(1,35)$ & $4,73(0,58)^{* *}$ & $3,19(0,23)^{* *}$ & 597,88 & 0,925 \\
\hline P. mucronata & $\beta_{0}$ & $\beta_{1}$ & $\boldsymbol{\beta}_{2}$ & $\mathbf{F}_{\text {calc }}$ & $\mathbf{R}_{\text {ajust. }}^{2}$ \\
\hline Área $=\hat{\mathrm{a}}_{0}+\hat{\mathrm{a}}_{1}(\mathrm{c})$ & $-29,44(1,30)$ & $8,39(0,18)^{\mathrm{ns}}$ & - & 2117,63 & 0,935 \\
\hline Área $=\beta_{0}+\beta_{1}(1)$ & $-21,62(1,08)$ & $9,60(0,20)^{* *}$ & - & 2335,00 & 0,940 \\
\hline Área $=\beta_{0}+\beta_{1}(\mathrm{cl})$ & $0,73(0,47)$ & $0,75(0,01)^{* *}$ & - & 4177,20 & 0,966 \\
\hline Área $=\beta_{0}+\hat{a}_{1}(c)+\beta_{2}(1)$ & $-27,04(0,96)$ & $4,12(0,38)^{* *}$ & $5,19(0,44)^{* *}$ & 2126,28 & 0,966 \\
\hline P. nitida & $\beta_{0}$ & $\beta_{1}$ & $\beta_{2}$ & $\mathbf{F}_{\text {calc }}$ & $\mathbf{R}_{\text {ajust. }}^{2}$ \\
\hline Área $=\beta_{0}+\beta_{1}(\mathrm{c})$ & $0,06(0,05)$ & $6,75(<0,01)^{* *}$ & - & 1619,05 & 0,917 \\
\hline Área $=\beta_{0}+\beta_{1}(1)$ & $0,05(0,04)$ & $3,95(<0,01)^{* *}$ & - & 2209,87 & 0,938 \\
\hline Área $=\beta_{0}+\beta_{1}(\mathrm{cl})$ & $1,17(0,01)$ & $0,79(<0,01)^{* *}$ & - & 11353,3 & 0,987 \\
\hline Área $=\beta_{0}+\beta_{1}(\mathrm{c})+\beta_{2}(1)$ & $-0,84(0,04)$ & $0,06(0,01)^{* *}$ & $0,12(0,01)^{* *}$ & 1561,72 & 0,955 \\
\hline P. setacea & $\beta_{0}$ & $\beta_{1}$ & $\beta_{2}$ & $\mathbf{F}_{\text {calc }}$ & $\mathbf{R}_{\text {ajust. }}^{2}$ \\
\hline Área $=\beta_{0}+\beta_{1}(\mathrm{C})$ & $-51,82(2,42)$ & $11,75(0,30)^{* *}$ & - & 1506,44 & 0,910 \\
\hline Área $=\beta_{0}+\beta_{1}(\mathrm{~L})$ & $-41,12(1,77)$ & $9,27(0,19)^{* *}$ & - & 2244,84 & 0,938 \\
\hline Área $=\beta_{0}+\beta_{1}(\mathrm{CL})$ & $-5,79(0,86)$ & $0,64(0,01)^{* *}$ & - & 3434,31 & 0,959 \\
\hline Área $=\beta_{0}+\beta_{1}(\mathrm{C})+\beta_{2}(\mathrm{~L})$ & $-45,92(2,00)$ & $3,76(0,86)^{* *}$ & $6,46(0,67)^{* *}$ & 1268,79 & 0,945 \\
\hline
\end{tabular}

** Significância de 0,$01 ;{ }^{\text {ns }}$ (não significativo); ${ }^{\text {a }}$ Erro padrão da estimativa. 

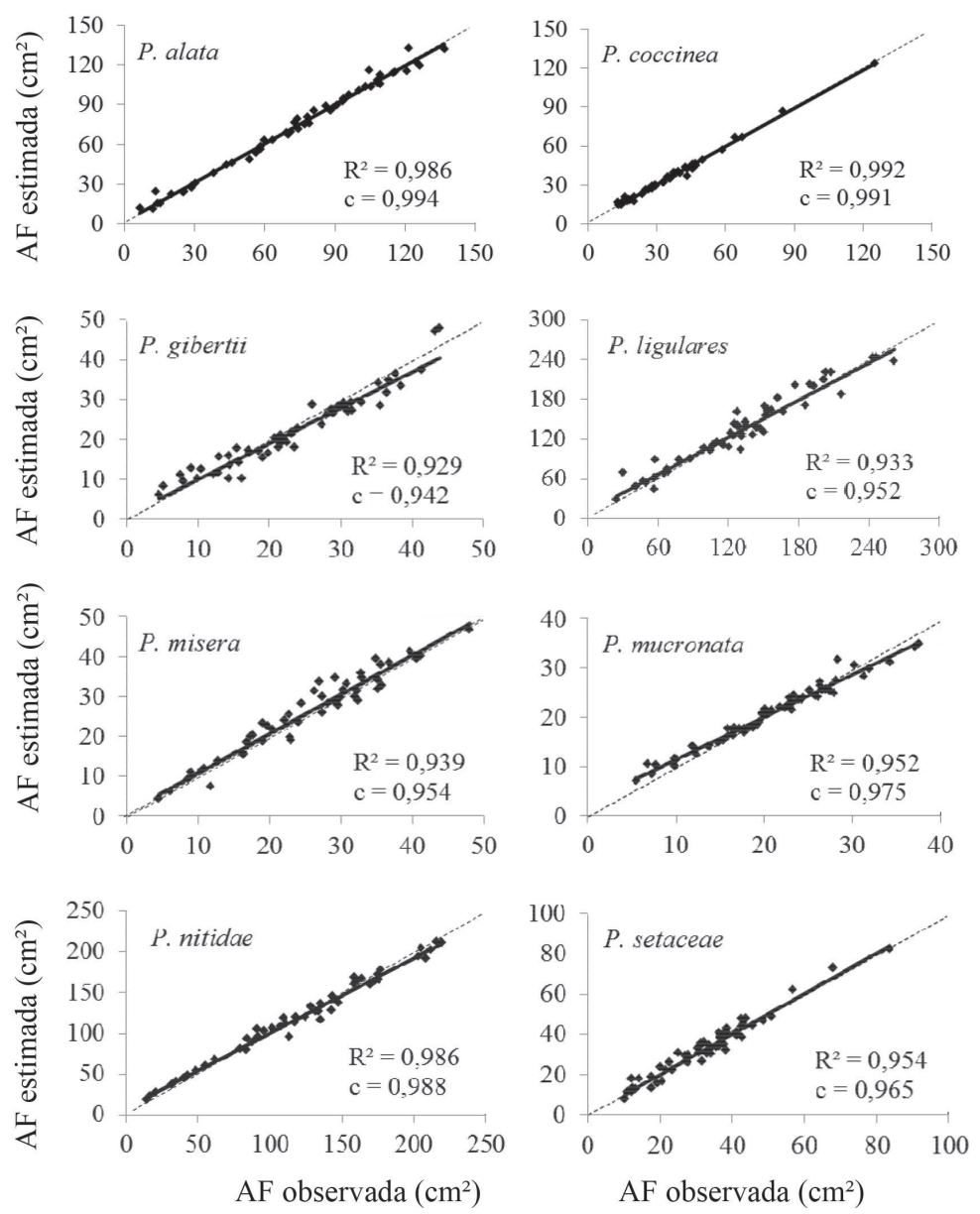

Figura 2. Relação entre a área foliar observada (AF observada) e a área foliar estimada (AF estimada), em $\mathrm{cm}^{2}$, e os índices estatísticos: coeficiente de determinação $\left(\mathrm{R}^{2}\right)$ e índice de confiança do modelo (c) para todas as espécies em estudo. A linha diagonal tracejada representa a reta 1:1.

sentaram ótimo desempenho, já que, para todas as espécies, o índice "c" foi superior a 0,942 (Figura 02).

A validação do modelo mostrou que a área foliar das espécies de Passiflora em estudo pode ser mensurada de forma rápida, precisa e não destrutiva, usando-se os modelos desenvolvidos.

De acordo com Campostrini \& Yamanishi (2001), os modelos matemáticos estimadores de área foliar para figueira podem ser obtidos por medições do comprimento das folhas das plantas, ao longo da nervura principal. A largura máxima foi considerada nos estudos com a cultura do meloeiro (Nascimento et al., 2002), do feijoeiro (Queiroga et al., 2003) e do feijão de porco (Toebe et al., 2012).

Entretanto, modelos, considerando o produto entre o comprimento e a largura do limbo foliar na estimação da área foliar, têm sido mais evidentes, entre os estudos relacionados, conforme os obtidos neste trabalho, para as diversas espécies de Passiflora, concordando com Bianco et al. (2008) e Pinto et al. (2008), que, em Sida cordifolia e Sida rhombifolia e em Curcuma alismatifolia e Curcuma zedoaria, respectivamente, obtiveram modelos mais precisos a partir do produto do comprimento vezes a largura do limbo das folhas.

\section{CONCLUSÕES}

A área foliar das espécies de Passiflora pode ser predita, usando-se simples medições lineares.

O produto do comprimento pela largura da folha é um parâmetro adequado para utilização como variável independente, na estimação da área foliar de maracujá, por meio de modelos de regressão.

\section{REFERÊNCIAS}

Ataíde EM, Oliveira JC \& Ruggiero C (2012) Florescimento e frutificação do maracujazeiro silvestre Passiflora setacea D. C. cultivado em Jaboticabal, SP. Revista Brasileira de Fruticultura, $34: 377-381$

Astegiano ED, Favaro JC \& Bouzo CA (2001) Estimacion del área foliar en distintos cultivares de tomate (Lycopersicon esculentum Mill) utilizando medidas foliares lineales. Investigation Agraria: Produccion y Proteccion Vegetales, 16:249-256. 
Blanco FF \& Folegatti MV (2005) Estimation of leaf area for greenhouse cucumber by linear measurements under salinity and grafting. Scientia Agricola, 62:305-309.

Bianco S, Carvalho LB \& Bianco MS (2008) Estimativa da área foliar de Sida cordifolia e Sida rhombifolia usando dimensões lineares do limbo foliar. Planta Daninha, 26:807-813.

Bianco S, Pitelli RA \& Pitelli AMCM (2003) Estimativa da área foliar de Typha latifolia usando dimensões lineares do limbo foliar. Planta Daninha, 21:257-261.

Camargo AP \& Sentelhas PC (1997) Avaliação do desempenho de diferentes métodos de estimativas da evapotranspiração potencial no Estado de São Paulo, Brasil. Revista Brasileira de Agrometeorologia, 5:89-97.

Campostrini E \& Yamanishi OK (2001) Estimation of papaya leaf area using the central vein length. Scientia Agricola, 58:3940 .

Cargnelutti Filho A, Toebe M, Burin C, Fick AL, Neu IMM \& Facco G (2012) Estimação da área foliar de mucuna cinza por meio de método não destrutivo. Ciência Rural, 42:238-242.

Centritto M, Loreto F, Massacci A, Pietrini F, Villani MC \& Zacchine M (2000) Improved growth and water use efficiency of cherry saplings under reduced light intensity. Ecological Research, 15:385-392.

Cho YY, Oh S, Oh MM \& Son JE (2007) Estimation of individual leaf area, fresh weight, and dry weight of hydroponically grown cucumbers (Cucumis sativus L.) using leaf length, width, and SPAD value. Horticultural Science, 111:330-334.

Demirsoy H, Demirsoy L \& Ozturk (2005) A. Improved model for the nondestructive estimation of strawberry leaf area. Fruits, 60:69-73.

Favarin JL, Dourado-Neto DY, García AG, Villa Nova NA \& Favarin MGGV (2002) Equações para a estimativa do índice de área foliar do cafeeiro. Pesquisa Agropecuária Brasileira, 37:769773.

Guo DP \& Sun YZ (2001) Estimation of leaf area of stem lettuce (Lactuca sativa var angustana) from linear measurements. Indian Journal of Agricultural, 71:483-486.

Jesus JRWC, Vale FXR, Coelho RR \& Costa LC (2001) Comparison of two methods for estimating leaf area index on common bean. Agronomy Journal, 93:989-991.
Lopes SJ, Brum B, Santos VJ, Faga EB, Luz GL \& Medeiros SLP (2007) Estimativa da área foliar de meloeiro em estádios fenológicos por fotos digitais. Ciência Rural, 37:1153-1156.

Lu HY, Lu CT, Wei ML \& Chan LF (2004) Comparison of different models for nondestructive leaf area estimation in taro. Agronomy Journal, 96:448-453.

Monteiro JEBA, Sentelhas PC, Chiavegato EJ, Guiselinei C, Santiago AV \& Prela A (2005) Estimação da área foliar do algodoeiro por meio de dimensões e massa das folhas. Bragantia.64:1524.

Nascimento B, Farias CHA, Silva MCC, Medeiros JF, Sobrinho JE \& Negreiros MZ (2002) Estimativa da área foliar do meloeiro. Horticultura Brasileira, 20:64-68.

Nesmith DS (1992) Estimating summer squash leaf area nondestructively. HortScience, 27:77.

Pinto ACR, Graziano TT, Barbosa JC \& Lasmar FB (2008) Modelos para estimativa da área foliar de Curcuma alismatifolia e Curcuma zedoaria. Bragantia, 67:549-552.

Queiroga JL, Romano EDU, Souza JRP \& Miglioranza E (2003) Estimativa da área foliar de feijão-vagem (Phaseolus vulgaris L.) por meio da largura máxima do folíolo central. Horticultura Brasileira, 21:64-68.

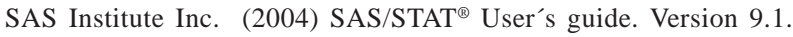
Cary, Statistical Analysis System Institute. 5121p.

Toebe M, Brum B, Lopes SJ, Cargnelutti Filho A \& Silveira TR (2010) Estimativa da área foliar de Crambe abyssinica por discos foliares e por fotos digitais. Ciência Rural, 40:445-448.

Toebe M, Cargnelutti Filho A, Burin C, Fick AL, Neu IMM, Casarotto G \& Alves BM (2012) Modelos para a estimação da área foliar de feijão de porco por dimensões foliares. Bragantia, 71:37-41.

Uzun S \& Çelik H (1999) Leaf area prediction models (uzc, elik-1) for different horticultural plants. Turkish Journal of Agriculture and Forestry, 23:645-650.

Willmott CJ, Ackleson SG, Davis RE, Feddema JJ, Klink KM, Legates DR, Rowe CM \& O'donnell J (1985) Statistics for the evaluation and comparison of models. Journal of Geophysical Research, 90:8995-9005. 